prevention of the rejection of some transplants. Mycophenolate mofetil was found to be associated with a lower risk of toxic adverse events such as ovarian failure, alopecia and leucopenia, compared with cyclophosphamide (1).

Objectives: To describe the population of patients with SLE receiving mycophenolate during a five year period.

Methods: We conducted a retrospective observational study, extracting information from a database in our rheumatic diseases center; we reviewed the clinical record of each patient with diagnosis of SLE and receiving mycophenolate. Descriptive epidemiology was performed for each variable presented.

Results: 1989 patients were diagnosed with Systemic Lupus Erythematosus (SLE) during 2011 and 2016 and 287 were receiving mycophenolate. Regarding demographic characteristics $94 \%$ were woman and $6 \%$ men, mean age was 49 years $\pm 15.41 \%$ of patients were employed, $40 \%$ were housekeepers, $11 \%$ students and $8 \%$ retired. The indication for mycophenolate was mainly for lupus nephritis $62 \%$, SLE in overlapping with systemic sclerosis $30 \%$ and only for SLE in $8 \%$ of cases. $36 \%$ of patients received a daily dose of $2000 \mathrm{mg}, 30 \% 1500 \mathrm{mg}$, $20 \% 1000 \mathrm{mg}, 8 \% 3000 \mathrm{mg}, 3.5 \% 4000 \mathrm{mg}$ and $1.5 \% 6000 \mathrm{mg}$ and $1 \% 500 \mathrm{mg}$. The mean value for 24 hour urine protein was $792 \mathrm{mg} / \mathrm{dl} \pm 140$ and for creatinine was $0.84 \mathrm{mg} / \mathrm{dl} \pm 0.36$. In our population patients were taking mycophenolate during a median of 24 months with a minimum of 6 and a maximum of 132 . In combination with mycophenolate $28 \%$ of the patients were taking corticoids, $21 \%$ hydroxichloroquine, $20 \%$ chloroquine, $17 \%$ antihypertensive drugs, $7 \%$ other medications and only $4 \%$ were taking the mycophenolate alone.

Conclusions: Despite of the absence of a license for mycophenolate for the management of Systemic Lupus Erythematosus, the use off-label of this drug continues to be frequently as an alternative and effective treatment in patients with lupus nephritis and other conditions associated to SLE.

References:

[1] National Institute for Health Care Excellence. NICE. Systemic lupus erythematosus: oral mycophenolate. England: NICE 2014

Disclosure of Interest: None declared

DOI: 10.1136/annrheumdis-2017-eular.5559

\section{AB0454 CLINICAL AND IMMUNOLOGICAL ACTIVITY IN POLISH COHORT OF SYSTEMIC LUPUS ERYTHEMATOSUS PATIENTS TREATED WITH GLUCOCORTICOIDS}

K. Pawlak-Bus, P. Leszczynski. Department of Rheumatology and Rehabilitation, University of Medical Sciences, Poznan, Poland

Background: Nowadays the lupus treatment strategy is based on background therapy, immunosuppressive drugs and glucocorticoids (GC). Using minimal effective dose of GC only in flares is a recomandation for preventing complications which increase mortality.

Objectives: To evaluate SLE clinical and immunological activity in lupus patients during the standard clinical care and analyze GC treatment

Methods: We observed Polish cohort of patients with SLE 127 patients (118 female and 9 male) with average age $43 \pm 6$ years, average disease duration $7,8 \pm 5,6$ years. All of them were treated with oral and pulse GC and standard immunosuppressive therapies (CTX, MMF, AZT,MTX, CsA). As a background therapy $77 \%$ of these patients were on chloroquine or hydroksychloroquine. All patients were assessed according to Systemic Lupus Erythematosus Disease Activity Index assessed by SLEDAl (version 2000) and divided into 5 groups: no GC, low dose, medium dose, high dose and puls GC therapy group. Immunological activity was assessed by anti-dsDNA and C3 and C4 complements levels.

Results: In analyzed group 28 of patients wihout GC the average SLEDAI score was 7 and $50 \%$ of this pts not revealed any immunological activity. Low dose of GC was used in 50 pts with average SLEDAl score 13 and in 24 pts of this group anti-dsDNA and C3 or C4 levels upper limit were not observed. Medium dose of GC was used in 20 pts with average SLEDAI score 19 and it was contained with high immunological activity in $55 \%$ ( $n=11$ ) of pts. In 27 of pts, high doses of GC including puls therapy were needed, the average SLEDAI score was very high 30 and most of pts from this group $70 \%$ were immunologicaly active.

Conclusions: In this Polish cohort lupus patients GC doses depended on lupus activity. Minimazing glucocorticoid exposure is an important part of appropriate management of lupus patients. Proper assessment of clinical and immunolgical lupus activity is critically for treatment decisions, especially for long-term GC use. Disclosure of Interest: None declared

DOI: 10.1136/annrheumdis-2017-eular.5400

\section{AB0455 SURVIVAL OF PREDNISONE-FREE REMISSION IN SLE PATIENTS WITH SEROLOGICALLY ACTIVE CLINICAL QUIESCENT DISEASE}

L. Nalotto, F. Ometto, M. Zen, L. laccarino, M. Gatto, M. Larosa, A. Doria. Department of Medicine, Rheumatology Unit, University of Padova (Italy), Padova, Italy

Objectives: To evaluate survival of prednisone (PDN) - free remission in systemic lupus erythematosus (SLE) patients and to investigate the potential predictors of disease flares.

Methods: Inclusion criteria were: (1) Diagnosis of SLE according to American College of Rheumatology (ACR) Classification Criteria of SLE; (2) Caucasian ethnicity; (3) Clinical remission (clinical SLEDAI-2K=0) at the time of PDNwithdrawal; (4) Stop of PDN treatment between 2010 and 2016; (5) At least two visits per year between January 2010 and April 2016.

Disease activity was assessed according to SLE Disease Activity Index-2000 (SLEDAI-2K). Damage was measured by the SLICC/American College of Rheumatology Damage Index (SDI). Flares were defined according to Safety of Estrogens in Lupus Erythematosus National Assessment (SELENA)-SLEDAI criteria.

We evaluated whether gender, age, age at PDN-stop, disease duration, duration of corticosteroid therapy, duration of remission before PDN withdrawal, time to flare, SLICC/American College of Rheumatology Damage Index (SDI) - score $>3$, positive anti-dsDNA antibodies (abs) and-or low C3/C4, type of SLE-involvement and concomitant immunosuppressive treatment could be predictors of flare. Multivariate logistic regression analysis was run to investigate the predictors of flare. Covariates included in the analysis were all variables reaching $p<0.20$ in the univariate analyses.

Results: Among 400 patients evaluated, 104 (26\%) fulfilled inclusion criteria Baseline characteristics are reported in table 1. Twenty-two $(21.2 \%)$ patients flared. Mean time to flare was $19.91 \pm 13.14$ months. Types of flare were 7 renal, 7 articular, 4 cutaneous, 2 haematological, 1 serositic and 1 neurological. Variables included in the multivariate logistic regression analysis were: positive anti-dsDNA abs and-or low $\mathrm{C} 3 / \mathrm{C} 4$, skin, articular and haematological involvement. Skin involvement resulted predictive of flare (OR $3.07,95 \% \mathrm{Cl} 1.11-8.53, \mathrm{P} 0.031)$ as reported in table 2 .

\begin{tabular}{|c|c|c|c|c|}
\hline & Aposents & 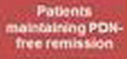 & 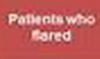 & D V owe \\
\hline Patents, $N$ & 105 & 22 & 22 & . \\
\hline Fenole, N P(s) & $91(87.5 \mathrm{~N})$ & $69(24,1 \%)$ & $229100005)$ & 0.048 \\
\hline Ade in 2019 , years, mesntso & $41.26=10.95$ & $49,46211.13$ & $40.45: 10,48$ & 0.723 \\
\hline Age st PoN stop. years, mesntsD & $3908 \times 11.2$ & $39.32: 11.27$ & $38.18: 10.63$ & 0.844 \\
\hline Discuse suretion moness, eseantso & $188,6421015 ?$ & 187649796 & 10204118.52 & 0.817 \\
\hline $\begin{array}{l}\text { Durabson ot corocosterotidnerapy, moens, } \\
\text { meantso }\end{array}$ & 13177,0274 & $12034 \times 53$ & $14073 \div 0816$ & 0.007 \\
\hline 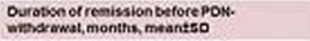 & $38.80=3501$ & $39.24=34.05$ & 31.91223 .51 & 0.785 \\
\hline 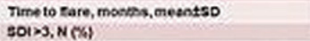 & mine & & $1291 \div 13.14$ & \\
\hline $301>3, N(4)$ & $100.8 \times 3$ & $8(285)$ & $2(21 \%$ & 0.325 \\
\hline 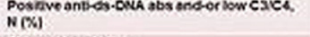 & $80(258 \times)$ & $\operatorname{cs}(822 x)$ & $21(055 \%)$ & 0.138 \\
\hline Skin roshea N (X) & $35(3737)$ & $22(2000)$ & 13(69.9) & 0.004 \\
\hline arthites, $N(x)$ & $69(602 \pi)$ & $51(022 \times 1)$ & $18(310 \%)$ & 0.004 \\
\hline Serosies, N(X) & $16(16.4 \%)$ & $13(15 \%)$ & $3(136)$ & 0.790 \\
\hline Okmerulonephriss, $N\left(y_{1}\right)$ & $65(62.5 \%)$ & $53(04.6 \%)$ & $12(545 \%)$ & 0.335 \\
\hline 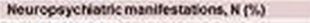 & $10(268)$ & $s(2855)$ & $2(218)$ & 0.025 \\
\hline Vascuibs, $N(x)$ & $6(5.85)$ & $4(49 x)$ & $2(2, \$)$ & 0.452 \\
\hline Hocmmologicolifmotivement, $N(x)$ & $25(240) 62$ & $17(20,7 \%)$ & $8(38,4 x)$ & 0.128 \\
\hline $\begin{array}{l}\text { Cansomitarkirmunosubpressivetreatemere, } \\
N(x)\end{array}$ & $41(29.4 \%)$ & $32(2900)$ & $9(10090)$ & 0.072 \\
\hline
\end{tabular}

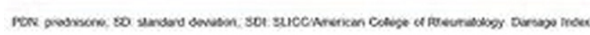

Risk of flare in prednisone-free remission, Logistic regression analysis

\begin{tabular}{|c|c|c|}
\hline & of (bx cil) & anist \\
\hline Skin involvereen & $205(1,11): 28$ & 0001 \\
\hline encuar isveoverwert & $275(0.90: 0<4)$ & 0.968 \\
\hline Meensological involverneat & $1.28(0.02573)$ & 0201 \\
\hline $\begin{array}{l}\text { Dounve anti-in CNA aba and or lon } \\
\text { caxc4 }\end{array}$ & $217(0.37 .2705)$ & a.28e \\
\hline
\end{tabular}

Conclusions: In SLE patients who stopped corticosteroid therapy, previous skin involvement resulted to be a predictor of disease flare.

Disclosure of Interest: None declared

DOI: 10.1136/annrheumdis-2017-eular.5925

\section{AB0456 SAFETY AND RETENTION RATE OF BELIMUMAB: DATA FROM A MULTICENTRIC ITALIAN STUDY}

L. laccarino ${ }^{1}$, M. Larosa ${ }^{1}$, L. Andreoli ${ }^{2}$, E. Bartoloni-Bocci ${ }^{3}$, A. Bortoluzzi $^{4}$, F. Ceccarelli ${ }^{5}$, F. Conti ${ }^{5}$, R. De Angelis ${ }^{6}$, G. De Marchi ${ }^{7}$, S. De Vita ${ }^{7}$, A. Di Matteo ${ }^{6}$, G. Emmi ${ }^{8}$, L. Emmi ${ }^{8}$, R. Gerli ${ }^{3}$, M. Gerosa ${ }^{9}$, M. Govoni ${ }^{4}$, M. Mosca ${ }^{10}$ P.L. Meroni ${ }^{9}$, G. Pazzola ${ }^{11}$, R. Reggia ${ }^{2}$, C. Salvarani ${ }^{11}$, C. Tani ${ }^{10}$, M. Zen ${ }^{1}$ A. Tincani ${ }^{2}$, A. Doria ${ }^{1} .{ }^{1}$ University of Padova, PADOVA; ${ }^{2}$ University of Brescia, BRESCIA; ${ }^{3}$ University of Perugia, PERUGIA; ${ }^{4}$ University of Ferrara, FERRARA; ${ }^{5}$ University la Sapienza, ROMA; ${ }^{6}$ University of Ancona, ANCONA $;{ }^{7}$ University of Udine, UDINE; ${ }^{8}$ University of Firenze, Firenze; ${ }^{9}$ University of Milano, MILANO

${ }^{10}$ University of Pisa, PISA; ${ }^{11}$ University of Reggio Emilia, Reggio Emilia, Italy

Background: Belimumab is used in the treatment of systemic lupus erithematosus (SLE), but few data on its safety in daily clinical practice are available to date. Objectives: To investigate safety, retention rate $(\mathrm{RR})$, reasons and predictors of belimumab discontinuation in a prospective multicentric Italian study.

Methods: A total of 188 active SLE patients refractory to standard therapy were treated with belimumab as add-on-therapy in 11 Italian centers. Adverse events (AEs) were defined as "any untoward medical occurrence in a patient 
treated with a pharmaceutical product which does not necessarily have a causal relationship with this treatment". AEs were subdivided in non-infectious, infectious, infusion and hypersensitivity reactions. Infusion and hypersensitivity reactions were defined as transient AEs related to belimumab occurring within 6 hours and 6-48 hours after drug administration, respectively. AEs was defined as severe (SAE) in case of hospitalization and/or death and/or life-threatening manifestations. Infections were considered severe in case of hospitalization and/or intravenous antibiotic use and/or death. Infusion and hypersensitivity reactions were considered severe when intensive care unit support was required. As baseline predictors of discontinuation the following variables were analyzed: gender, age, age at SLE onset, disease duration, disease activity pattern (relapsing remitting or chronic active), SLEDAI-2K $\geq 10$, prednisone $>7.5 \mathrm{mg} /$ day, concomitant immunosuppressant, antimalarial drug use, number and type of comorbidities, number of previous organ involvement, type of major involvement and number of flares in the 12 months before belimumab initiation. Data were analyzed using the SPSS (version 23.0, Chicago, IL) software.

Results: A total of 453 EAs were recorded in 132 patients after a mean follow-up period of $17.5 \pm 10.6$ months (range 3-36): 443 (97.8\%) were non severe and 10 (2.2\%) SAEs (Table 1). No deaths and severe infusion/hypersensitivity reactions occurred. Belimumab discontinuation was observed in 58 patients $(30.8 \%)$ after $10.4 \pm 7.5$ months of follow-up (Table 2). RR was $91.5 \%$ at 6 months, $81.4 \%$ at 12 months, $72.9 \%$ at 18 months, $72.4 \%$ at 24 months, $69.7 \%$ at 30 months and $69.2 \%$ at 36 months. No associations were found between baseline variables and drug discontinuation.

Table 1. AEs observed in 188 patients with refractory SLE treated with belimumab Ptswith AEs (N) Pts with AEs (\%) AEs (N) AEs (\%

\begin{tabular}{lcccc} 
Non severe AEs & 129 & 68.6 & 443 & 97.8 \\
Infectious AEs & 101 & 53.7 & 273 & 60.3 \\
Non infectious AEs & 57 & 30.3 & 84 & 18.5 \\
Hypersensitivityreactions & 26 & 13.8 & 81 & 17.9 \\
Infusion reactions & 4 & 2.1 & 5 & 1.1 \\
SAEs & 9 & 4.8 & 10 & 2.2 \\
Infectious SAEs & 1 & 0.5 & 1 & 0.2 \\
Non infectious SEAs & 8 & 4.3 & 9 & 2.0 \\
\hline Total & 132 & 70.2 & 453 & $\mathbf{1 0 0 . 0}$ \\
\hline
\end{tabular}

Pts: patients; AEs: acherse events; SAEs: severe acherse events.

Table 2. Reasons of discontinuation of belimumab

\begin{tabular}{lcc} 
& Pts (N) & Pts (\%) \\
AEs & 22 & 37.9 \\
Non-responder (articular/renal cutaneous haematological) & 16 & 27.6 \\
Pregnancy & 8 & 13.8 \\
Flare (renal/articular) & 6 & 10.3 \\
Lost of follow-up & 4 & 6.9 \\
Remission & 2 & 3.4 \\
Total & $\mathbf{5 8}$ & $\mathbf{1 0 0 . 0}$ \\
\hline Pts: potients. & &
\end{tabular}

Conclusions: Belimumab demonstrated a good safety profile with a low rate of SAEs. Discontinuation occurred in less than $1 / 3$ of subjects with a low rate of discontinuation due to AEs.

Disclosure of Interest: None declared

DOI: 10.1136/annrheumdis-2017-eular.6088

\section{AB0457 LONG-TERM EFFECTIVENESS OF TREATMENTS FOR NEUROPSYCHIATRIC MANIFESTATIONS OF SYSTEMIC LUPUS ERYTHEMATOSUS}

\section{L.D. Fajardo Hermosillo. Rheumatology, IMSS, Guadalajara, Mexico}

Background: Neuropsychiatric involvement of systemic lupus erythematosus (NPSLE) is one of the most deleterious complications of the disease, leading to great decrease of quality of life and poor prognosis [1]. Factors such as activity of disease have been associated NPSLE [1,2]. The current treatment of NPSLE remains mostly empiric, requiring high-dose corticosteroids and extensive immunosuppressive therapy $[3,4]$. Short-term efficacy of treatments in NPSLE have been evaluated by several studies, however few studies have been performed to evaluate long-term efficacy of these drugs [4].

Objectives: To compare the long-term effectiveness among drugs and associated factors in the treatment of NPSLE.

Methods: 209 patients ( $\geq 14$ years) from a Mexican cohort from 2011 to 2017 were examined. SLE and NPSLE cases fulfilled 1997 ACR and 1999 ACR criteria respectively. Demographic factors, comorbidities and pharmacologic treatments were reviewed for patients with NPSLE. Variables were studied by bivariate, multivariate and survival analyses.

Results: Of 209 SLE patients, 37 (17.7\%) had NPSLE; of them 32 were women. The mean age [standard deviation (SD)] was 38.8 (14.6) years. The mean of time at onset of SLE (SD) was 6.2 (5.4) years. SLEDAI and SLICC mean (SD) were $22.1(10.2)$ and $1.83(1.2)$ separately. Diffuse and focal manifestations of NPSLE were presented in 20 and 17 cases. Central and peripheral nervous system events of NPSLE were described in 34 and 3 of patients. The NPSLE manifestations more prevalent were cerebrovascular disease $(n=15)$, psychosis $(n=8)$ and seizure disorders $(n=5)$. A total of 112 cycles of treatment were analyzed [prednisone (PDN) $n=30$, intravenous (iv) methylprednisolone (MTP) $n=23$, iv cyclophosphamide (CYC) $n=13$, azathioprine (AZA) $n=12$, mycophenolate mofetil (MMF) $n=11$, rituximab (RTX) $n=10$, hydroxichloroquine (HCQ) $n=9$, plasmapheresis (PPH) $n=2$ and iv immunoglobulin (IVIg) $n=2$ ]. Cognitive dysfunction was more associated to higher SLE activity, damage and mortality. Also, psychosis was more associated to receive higher doses of oral PDN or iv MTP, to employ iv CYC and to be linked with renal SLE disease activity. On the other hand, cerebral vascular disease was more associated to receive $\mathrm{HCQ}$ and to have antiphospholipid antibodies. The main cause to therapy discontinuation was inefficacy and was more common in patients treated with iv CYC. Corticosteroids survival in months was higher in-group PDN $<15$ $\mathrm{mg} / \mathrm{d}(97.89 \pm 16.4$; IC95\% 65.8-130). AZA survival in months $(90 \pm 30.8$; IC95\% 29.6-150) was higher than others treatments for NPSLE and was more associated to use IVIg (OR 10.8, 95\% Cl 1.04-111, $\mathrm{p}=0.04$ ). When the first therapy was failed, the drug as second therapy used with higher survival in months was MMF

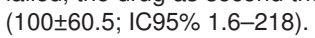

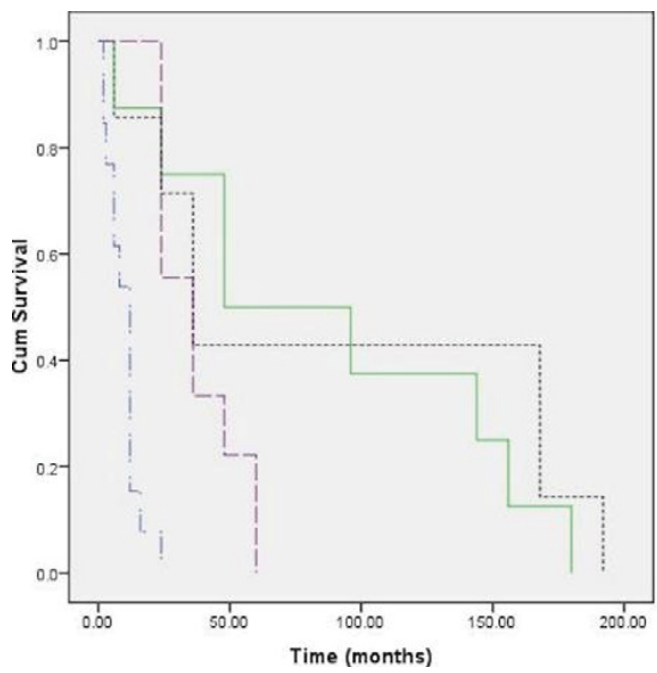

Treatments $\sim$ NAZA $\rightarrow$ TMUF

Conclusions: This study suggests that patients with diffuse manifestations and central nervous system involvement in NPSLE presented more activity, damage and mortality and therefore used more PDN, MTP and CYC. Among the treatments as first therapy for NPSLE with the better long-term efficacy was AZA and when it failed, MMF remained as better second therapy.

\section{References:}

[1] Arthritis Rheum 1999;42:599-608.

[2] Semin Arhtritis Rheum 2011;41;1-11.

[3] Ann Rheum Dis 2010;69(3):529-535.

[4] CNS DRugs 2011;25(9):721-736.

Disclosure of Interest: None declared

DOI: 10.1136/annrheumdis-2017-eular.2815

\section{AB0458 B CELLS DEPLETION FOR THE TREATMENT OF SYSTEMIC AUTOIMMUNE DISEASES}

M.L. Velloso Feijoo, S. Rodriguez Montero, N. Plaza Aulestia, J.L. Marenco de la Fuente. Rheumatology Unit, Valme University Hospital, Seville, Spain

Background: Systemic autoimmune diseases (SAD) have traditionally been treated with steroids and immunosuppressants, but not all patients respond to these measures.

Rituximab (RTX) has been used in several SAD with favorable results, but there are only a few reports of isolated clinical experiences, with a very small number of patients.

Data about use of this drug under conditions of daily clinical practice may be relevant.

Objectives: To describe the characteristics of patients with SAD who are candidates for treatment with RTX.

To evaluate the response to this drug in the different SAD.

Methods: Demographic data and disease, treatment and response variables were included.

We use the EULAR definitions of partial response (improvement of at least $50 \%$ of the main manifestations of the disease) and complete response (disappearance of the manifestations of the disease), because of the heterogeneity of the SAD and their multiple manifestations.

Results: We included 53 patients, 90.6\% were women. The mean age at diagnosis was $31.42 \pm 14.33$ years; and the median duration of disease at the onset of RTX $1.99(0-7.5)$ years. Patients received a median of 2 cycles (1-3 min 